\title{
Development of a Parametric Program for Processing a Hyperbolic Surface on a Lathe Machine
}

\author{
Tsvetan Kaldashev \\ Technical university of Sofia \\ Sofia, Bulgaria \\ email: kaldashev.cvetan@abv.bg
}

\begin{abstract}
This paper discusses the development of a parametric (macro) program for processing a hyperbolic surface on a CNC lathe machine. Analytical dependences have been used to develop the macro program, as a result of which the CNC (computer numerical control) automatically calculates the coordinates of the intermediate points of the tool trajectory. The WHILE cycle operator is used to run the tool. Macro program functionality has been verified in a virtual environment using Vericut.
\end{abstract}

Keywords-CNC, hyperbola, parametric (macro) program.

\section{INTRODUCTION}

Parametric programming, in addition to $\mathrm{CNC}$ commands and functions, also involves the use of variables, arithmetic actions, trigonometric and other functions, algorithmic structure building, system variables, CNC external linking and control devices "to" and "from" via interface variables [4]. This considerably expands the capabilities of CNC machines by allowing the development of parametric programs for processing of typical surfaces and the creation of a technological library, significantly simplified programs for approximating movements on curves other than a circle (and in general different from those in the basic language), which have an analytical description and others. Variables, as in any algorithmic language, are used to store and subsequently use the values that are changing in the course of the program. Using variables makes the program more flexible than using ordinary subroutines. The variables are identified by their name, formed by the "hash mark" - \#, followed by a number or a formula. The variables are user and system variables (Table 1). The first set of variables (user) can be used by the developer at random, and the second group (system) represents fixed-term variables, most commonly related to the $\mathrm{CNC}$ system job records. When constructing algorithmic structures, the GOTO unconditional transition operators, conditional IF operator and WHILE [1] cycle operator are used.
TABLE I.

\begin{tabular}{|c|c|l|}
\hline Type of variables & Function & Designation \\
\hline \multirow{3}{*}{ User variables } & Local & $\# 1 \div \# 33$ \\
\cline { 2 - 3 } & Common & $\# 100 \div \# 149$ \\
& & $\# 500 \div \# 509$ \\
\hline \multirow{5}{*}{ System variables } & with special purpose & $\# 1000 \div \# 1015$ \\
& & $\# 1100 \div \# 1115$ \\
& & $\# 1000 \div \# 1015$ \\
& & $\# 3032 \div \# 1132$ \\
& & $\# 30003 \div \# 3005$ \\
& & $\# 4001 \div \# 4120$ \\
& & $\# 5 \times x x$ \\
\hline
\end{tabular}

II. PARAMETRIC PROGRAM FOR PROCESSING HYPERBOLIC SURFACE

The need of developing a parametric program for treating a hyperbolic surface results from the fact that rolling rolls are required to be processed for rolled periodically. In the process of employment, the hyperbolic surface is worn out and its profile deviates from the set, which affects the end product.

Hyperbola is a planar curve of second order and consists of two branches having two focuses (f1 and f2) and asymptotes [3] (Figure 1). The asymptotic intersection is a center of symmetry for the hyperbola. The axis of the hyperbola, called the main axis, coincides with the x-axis. Its vertices have coordinates $(a, 0)$ and $(-a, 0)$. Parametric equations of the hyperbolic branch corresponding to $\mathrm{x}>$ 0 are:

$$
\begin{gathered}
X=\frac{\alpha}{\operatorname{coc}(\alpha)} \\
Y=b-\tan (\alpha)
\end{gathered}
$$

As a is the length of the main axis coinciding with the axis $\mathrm{x}$ (Figure 1), and e is the length of the minor axis coinciding with the $y$ axis. 


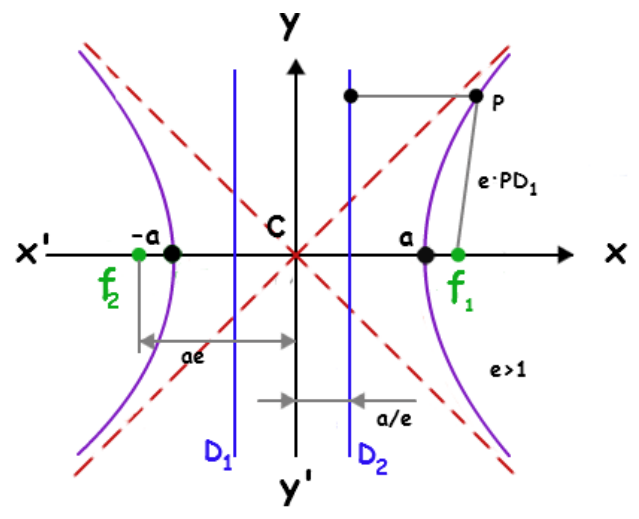

Fig. 1. Hyperbola [2]

The processing of a hyperbolic surface can be accomplished using a CAD / CAM system, where a 3D model is created in advance, from which the control programs are generated. In figure 2 is shown a window where the parametric equation of hyperbola is recorded, as a result of which the system automatically constructs the curve.

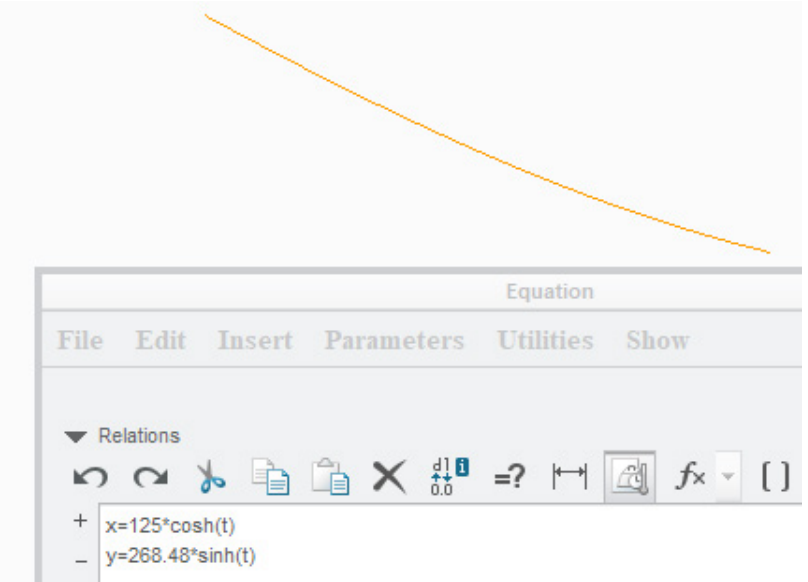

Fig. 2. Construction of hyperbola in parametric equation using the tool Equation

The PTC Creo CAD / CAM system uses the Equation tool. For curve construction is used parametric equation of hyperbola with hyperbolic functions.

After the construction of the hyperbolic curve using the Revolve tool, the CAD model of the workpiece is created. Figure 3 shows the final 3D model.

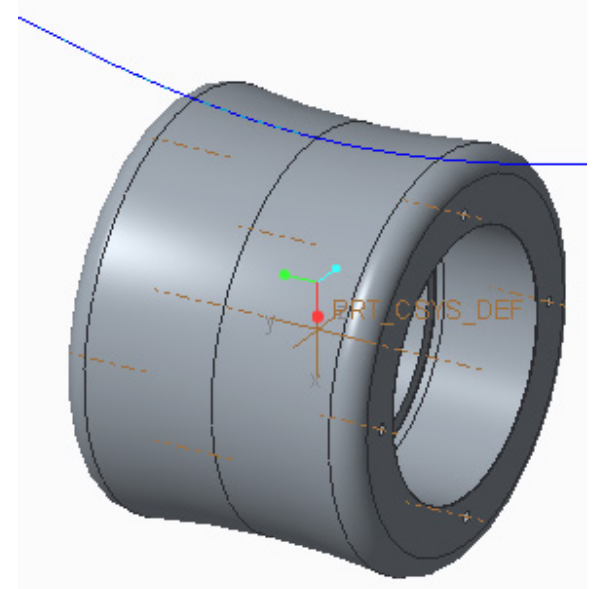

Fig. 3. 3D model of detail

The development of instrumentation transitions for the treatment of the hyperbolic surface is carried out in the CAM environment of PTC Creo. Figure 4 shows the clean instrumental transition for the workpiece. As already mentioned, the $\mathrm{CNC}$ does not have a second-order surface interpolator, which means that the curve should be approximated with straight lines.

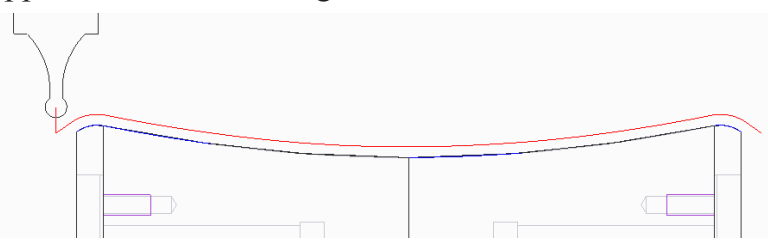

Fig. 4. Tool path

Accuracy can be controlled with the Tolerance parameter so that the hyperbolic surface of the workpiece is processed with the least possible deviation from the curve. This means that the smaller the value set in the Tolerance parameter, the deviation from the curve will be less, but on the other hand it will affect the volume of the control program - it will increase.

In order to avoid the aforementioned processing problems, a good solution is to develop a parametric program, thus the $\mathrm{CNC}$ will automatically calculate the coordinates of the workpiece contour with precision to the $\mathrm{CNC}$ input increment. The program has the algorithm shown in figure 5.

As the input data, the main axle length coinciding the $X$ axis (\#100), the length of the minor axis (\#101), in the case coinciding with the $\mathrm{Z}$ axis, the increment of the angle reduction (\#102), the initial (\#103) and the final (\#104) angles associated with the hyperbola treatment and the overall length of the workpiece (\# 108).

In order to be able to process the workpiece it is necessary to have the working coordinate system positioned symmetrically in relation to its length (Figure 6). Therefore, two portions of the hyperbola are conventionally indicated in figure 6 with first and second stretches. Each of the plots is L/ 2 long. 


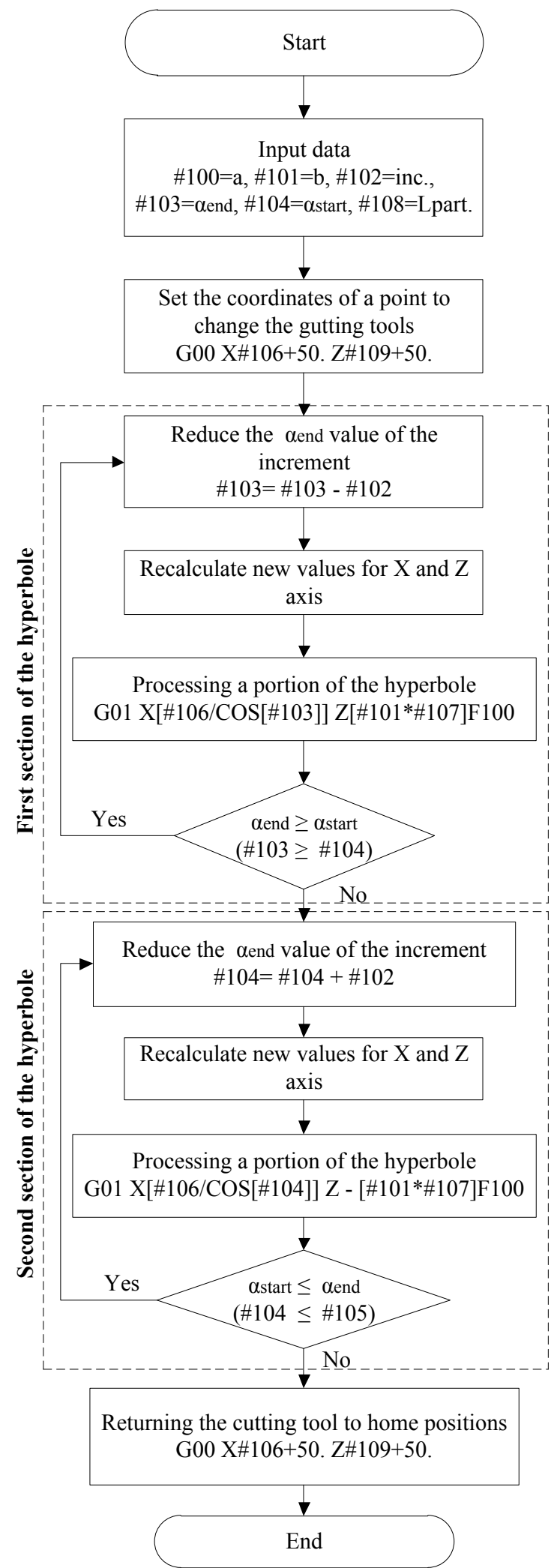

Fig. 5. Block diagram of the parametric (macro) program

Parametric equation (1) of the hyperbola is used in developing the parametric program. Two operators are used for the WHILE cycle, the first being used to handle the first section of the hyperbola and the second for the second section of the hyperbola.

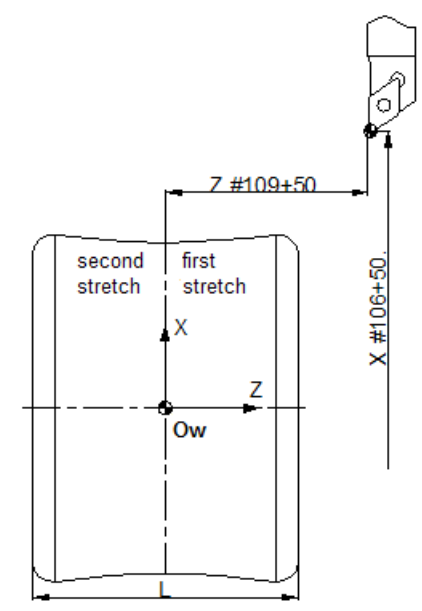

Fig. 6. Annotation of some variables used in the parametric program The parameter program has the following structure:

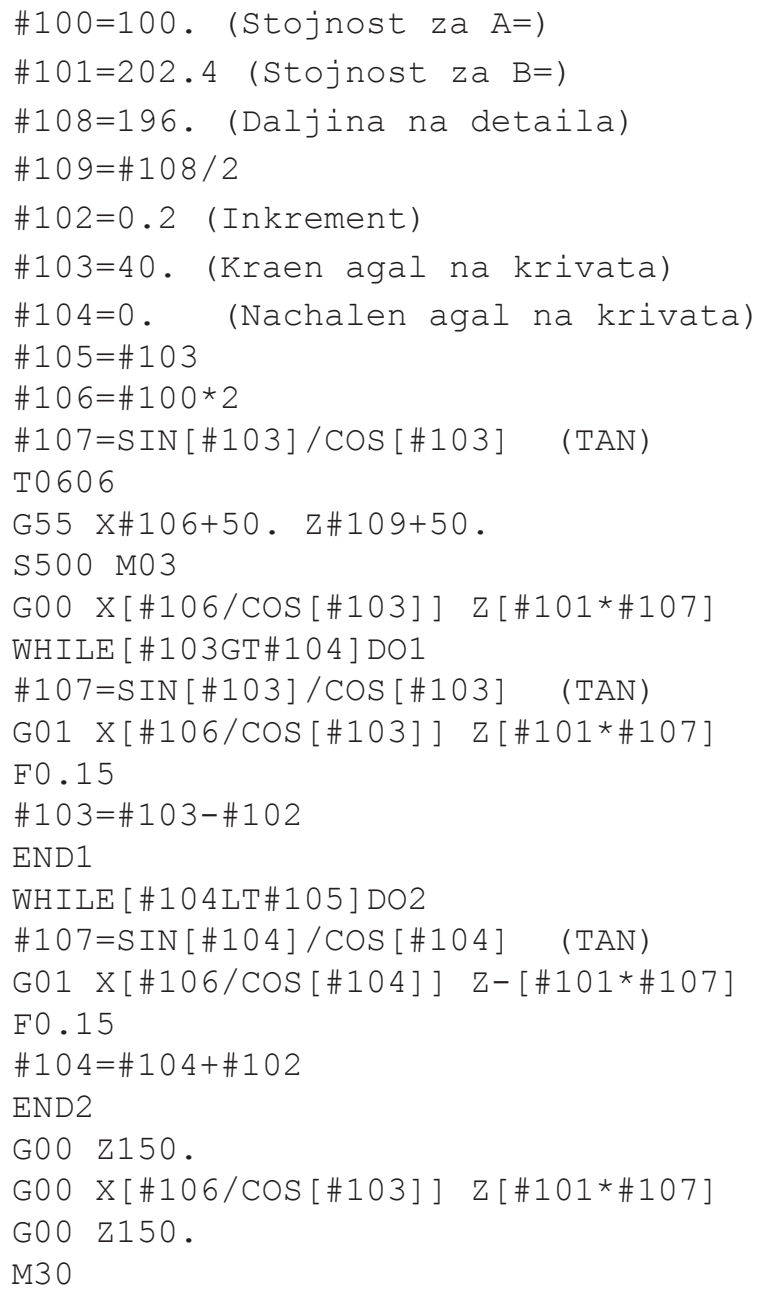

The parametric program was verified in a virtual environment using Vericut. In figure 7 is a screen of the simulation with the developed macro program.

In order to assess whether it is correct, if there is a deviation of the surface from the theoretical examination, the AutoDiff tool is used. It is used to automatically detect the differences between the resulting model as a result of the simulation and the one generated by the control programs. As a result of the analysis no difference was found (Figure 8 - No Differences) from where it can be concluded that the macro program is efficient. 


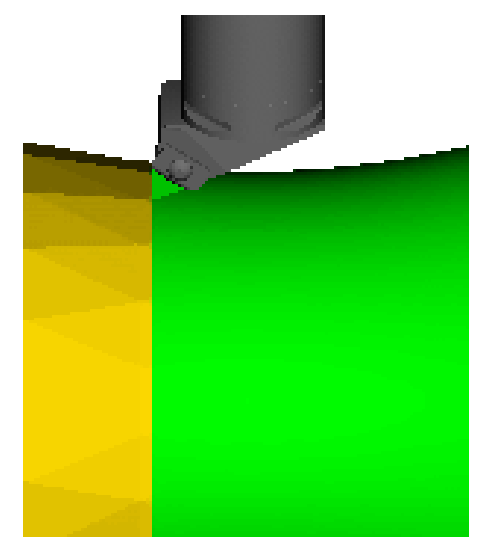

\section{CONCLUSION}

The parametric program developed has been verified in a Vericut virtual environment. An analysis of the AutoDiff tool has been made from which it can be concluded that the program is work efficient.

\section{REFERENCES}

[1] Ben Groves, Macro B programming manual

[2] www.tutorvista.com, visited on 30.07.2018 г.

[3] www.wikipedia.org, visited on 30.07.2018 г.

[4] Hadjiiski P., Programming of CNC machine tools, TU-Sofia, 2010 г., ISBN 978-954-438-865-2

Fig. 7. Simulation in Vericut of the parametric program developed

\section{(7) AUTO-DIFF}

\begin{tabular}{|c|c|c|c|c|}
\hline Settings & Options & Compare & By Region & \\
\hline Stock & Componer & & Stock & $\checkmark$ \\
\hline Desigr & Compon & ent & Design & $\checkmark$ \\
\hline$\square$ Sto & ck Display & & Solid & $\checkmark$ \\
\hline$\square \mathrm{De}$ & sign Displ & & Solid & $\checkmark$ \\
\hline Comp & rison Met & hod & Profile & $\checkmark$ \\
\hline Comp & rison Typ & & Excess & $\checkmark$ \\
\hline on Tolerar & & & & \\
\hline Excess & & & & \\
\hline 0,4 & & 42:Royal B & Bue & I \\
\hline & (c) & No Differ & ences & \\
\hline Apply & Compare & Restore & Report... & Close \\
\hline
\end{tabular}

Fig. 8. Analysis with the AutoDiff tool $\times$ 